\title{
Pink Breast Milk: Serratia marcescens Colonization
}

\author{
Cipatli Ayuzo del Valle, MD ${ }^{1}$ Emilio Treviño Salinas, MD, $\mathrm{PhD}^{2}$ \\ ${ }^{1}$ Colegio de Pediatría de Nuevo León México, University of Nuevo Leon \\ Mexico, Monterrey, Nuevo León, Mexico \\ 2 Division of Gynecologic Oncology, University of Nuevo Leon Mexico, \\ Monterrey, Nuevo León, Mexico \\ Address for correspondence Cipatli Ayuzo del Valle, MD, Centro \\ Medico Conchita, 15 de Mayo 1822 Colonia María Luisa, Monterrey, \\ Nuevo León, Mexico (e-mail: cipatlimd@yahoo.com).
}

Am J Perinatol Rep 2014;4:e101-e104.

\begin{abstract}
Keywords

- breastfeeding

- colonization

- enterobacteria

Background Breast milk can turn pink with Serratia marcescens colonization, this bacterium has been associated with several diseases and even death. It is seen most commonly in the intensive care settings. Discoloration of the breast milk can lead to premature termination of nursing. We describe two cases of pink-colored breast milk in which S. marsescens was isolated from both the expressed breast milk. Antimicrobial treatment was administered to the mothers. Return to breastfeeding was successful in both the cases.

Conclusions Pink breast milk is caused by S. marsescens colonization. In such cases, early recognition and treatment before the development of infection is recommended to return to breastfeeding.
\end{abstract}

Breast milk can turn into a pinkish color due to colonization by Serratia marcescens, a species of rod-shaped gram-negative bacteria that produce a reddish-orange tripyrrole pigment called prodigiosin ${ }^{1}$ that has been related to a variety of diseases and even newborn deaths.

We describe two cases of pink colored breast milk in which S. marsescens was isolated from both the samples.

\section{Cases}

\section{Case 1}

A 29-year-old primigravid woman underwent a normal vaginal delivery at 37.5 weeks gestation. A healthy baby girl was delivered that weighed $2.7 \mathrm{~kg}$. Both mother and the baby were discharged home. One week postpartum, her baby was readmitted for phototherapy.

At her 5th week of checkup she reported a bright pink coloration of cloth towels used for breastfeeding ( - Fig. 1). She denied fever, mastitis, nipple trauma or any other pertinent symptom. However, despite her baby presenting normothermic, the mother reported poor oral intake. The mom was treated with ciprofloxacin empirically. Culture from the expressed breast milk showed several colonies of S. marcescens, in addition, Enterobacteria spp. was isolated from the infant's oropharynx that was then treated with trimetoprim and sulfametoxazol. Stool and urine cultures were negative. She was advised to pump and discard the milk while using antibiotics. Repeat cultures were negative and she returned to breastfeeding without any incident.

\section{Case 2}

A 33-year-old woman chose to exclusively breastfeed her infant. The male infant was delivered at term by an elective cesarean section. There were no complications associated with the pregnancy and delivery. Ten weeks postpartum, she noticed pink stains on cloth towels used during breastfeeding (-Fig. 2). Breast pump was never used and there was no nipple trauma reported. The baby was closely monitored with no signs or symptoms noted on the history and physical examination.

Cultures of the mother's breast milk tested positive for S. marcescens, henceforth she was subsequently treated with cephalosporin. The baby's cultures were all negative. She returned to breastfeeding exclusively as soon as the breast milk culture was reported negative (-Fig. $\mathbf{3}$ ).

\section{Discussion}

S. marcescens is a gram-negative bacillus in the Enterobacteriaceae family. Several outbreaks have been linked to received

December 23, 2013

accepted after revision

June 25, 2014

published online

November 18, 2014
DOI http://dx.doi.org/

10.1055/s-0034-1387934. ISSN 2157-6998.
Copyright (c) 2014 by Thieme Medical Publishers, Inc., 333 Seventh Avenue, New York, NY 10001, USA. Tel: +1(212) 584-4662.
License terms

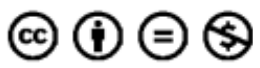




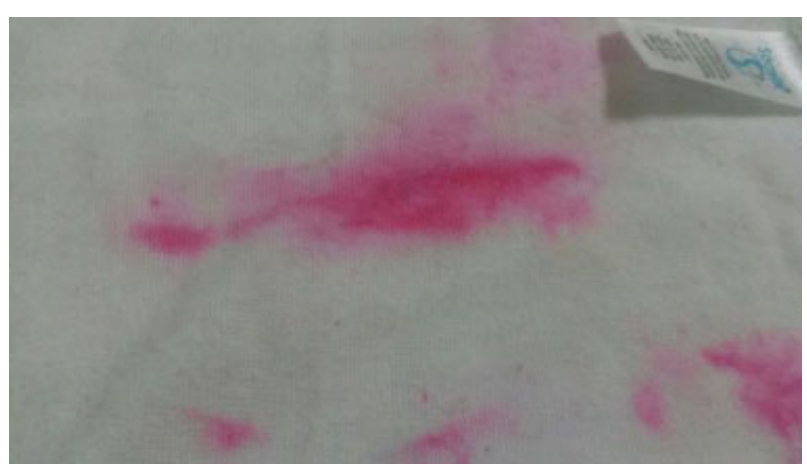

Fig. 1 Pink stains on the cloth towel due to Serratia marcescens colonization.

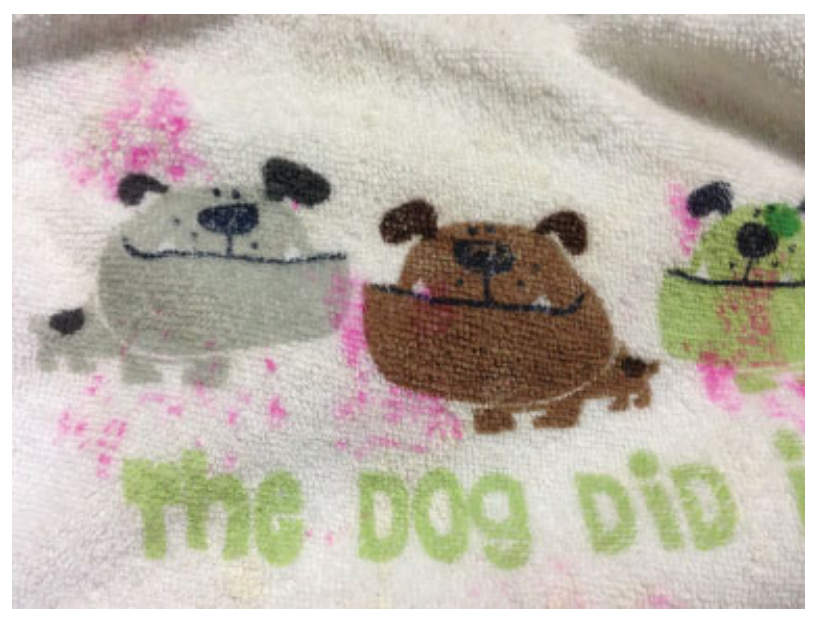

Fig. 2 Serratia marcescens pink stains on the cloth towel.

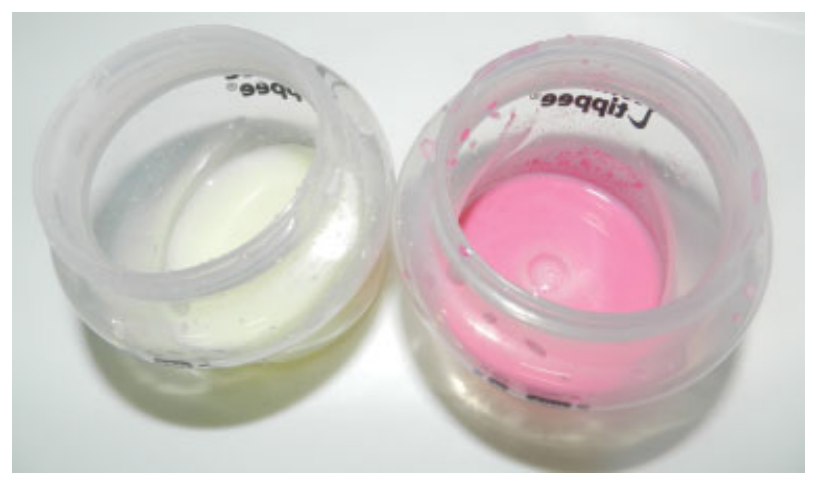

Fig. 3 Expressed breast milk after and before antibiotic treatment.

contaminated medical equipment, improper hand hygiene by health care workers and breast milk. ${ }^{2}$ S. marcescens species appear to be common environmental organisms, which helps to explain the large number of nosocomial infections due to this bacterium. In the literature, there have been a very large number of reported hospital-related S. marcescens outbreaks. Because there are so many described hospital- associated outbreaks, it is often assumed that infections caused by Serratia are primarily nosocomial in origin. ${ }^{3}$ Recently however,
Laupland et al conducted an extensive survey of $S$. marcescens infections in Canada and found that $65 \%$ of all infections caused by $S$. marcescens were community based. ${ }^{4} S$. marcescens produces a characteristic brightly colored pigment, prodigiosin, that was once used as a tracer organism by investigators in medical fields and even in combat zones. ${ }^{3}$

The first time S. marcescens was described in cloth towels was in 1958 by Waisman and Stone, who described the "red diaper syndrome," the appearance of $S$. marcescens in soiled diapers of a female baby born in Wisconsin. The parents noticed that soiled diapers that have been rinsed with plain water turned red. The stool of the infant was cultured and S. marcescens was recovered. Although, the baby never had signs or symptoms or illness, physicians treated her with oral sulfasuxidine. $^{5}$

A survey from 1997 data on SENTRY Antimicrobial Surveillance Program isolates from the United States, Canada, and Latin America showed that Serratia species were the 12th most common organisms associated with bloodstream infections. ${ }^{6}$

Although, the actual number of organisms excreted in milk is unknown, it is unlikely that an infant taking milk directly from his mother's breast will ingest enough organisms to cause disease. However, improper handling and storage of milk may enable organisms/pathogens to multiply to numbers sufficient to cause disease, especially in infants at higher risk for infection, such as those born preterm. Contamination with $>1,000$ gram-negative bacilli per milliliter is associated with feeding intolerance, whereas higher levels of contamination $(>1,000,000 / \mathrm{mL})$ can be associated with sepsis. ${ }^{7}$

In the two cases that we have presented there were no breast pumps associated with infections, even though several cases have been described previously in the literature. ${ }^{2,8,9}$ Even though our first case could have been infected on her 4th day of hospitalization, the second case could have been community acquired as described by Laupland et al. ${ }^{4}$

Although, there are no clear recommendations for women with $S$. marcescens colonization, and due to high risk of sepsis associated with this bacteria, treatment with antibiotics is highly recommended. Returning to breastfeeding is safe after cultures of both the mother and the baby are negative.

\section{Conflict of Interest}

The authors declare no conflict of interest.

\section{References}

1 Bennett JW, Bentley R. Seeing red: the story of prodigiosin. Adv Appl Microbiol 2000;47:1-32

2 Macdonald TM, Langley JM, Mailman T, et al. Serratia marcescens outbreak in a neonatal intensive care unit related to the exit port of an oscillator. Pediatr Crit Care Med 2011;12(6):e282-e286

3 Mahlen SD. Serratia infections: from military experiments to current practice. Clin Microbiol Rev 2011;24(4):755-791

4 Laupland KB, Parkins MD, Gregson DB, Church DL, Ross T, Pitout JD. Population-based laboratory surveillance for Serratia species isolates in a large Canadian health region. Eur J Clin Microbiol Infect Dis 2008;27(2):89-95 
5 Waisman HA, Stone WH. The presence of Serratia marcescens as the predominating organism in the intestinal tract of the newborn; the occurrence of the red diaper syndrome. Pediatrics 1958; 21(1):8-12

6 Diekema DJ, Pfaller MA, Jones RN, et al. Survey of bloodstream infections due to gram-negative bacilli: frequency of occurrence and antimicrobial susceptibility of isolates collected in the United States, Canada, and Latin America for the SENTRY Antimicrobial Surveillance Program, 1997. Clin Infect Dis 1999;29(3):595-607
7 Youssef RF, Darcy E, Barone A, Borja MT, Leggiadro RJ. Expressed breast milk as a source of neonatal sepsis. Pediatr Infect Dis J 2002; 21(9):888-889

8 Faro J, Katz A, Berens P, Ross PJ. Premature termination of nursing secondary to Serratia marcescens breast pump contamination. Obstet Gynecol 2011;117(2 Pt 2):485-486

9 Villa J, Alba C, Barrado L, et al. Long-term evolution of multiple outbreaks of Serratia marcescens bacteremia in a neonatal intensive care unit. Pediatr Infect Dis J 2012;31(12):1298-1300 